\title{
Application of Fractal Geometry in Computer Hosiery Machine
}

\author{
Zhang Rui ${ }^{1, a^{*}}$, Jiang ZhiWei ${ }^{1, b}$ \\ ${ }^{1}$ College of Information Science and Techology, Zhejiang Shuren University, Hangzhou, Zhejiang, \\ 310015, China \\ a396107172@qq.com, bjzw219@126.com
}

Keywords: fractal picture; hosiery machine pattern; save pattern

\begin{abstract}
The shortage of China hosiery machine patterns is analyzed ,Normal models, such as iterated function system, recursion fractal, complex plane fractal system, and cellular automata, integrated with Visual c++'s excellent performance in graphics are utilized to draw and obtain various designs based on the object-oriented technique. The fractal patterns are saved as bmp pictures. The signal data may be downloaded to the controller, and the pattern's height and width can be used to control the hosiery machine to weave numerous patterns. This work is valuable to enhance the CAD level of sock pattern design and productivity of a computerized hosiery machine.
\end{abstract}

\section{Introduction}

In modern society, socks are not only a cloth material used by people to keep warm but also a soft material for decoration and beautification in daily life [1]. Therefore, pattern design in socks plays an important role in a consumer's decision to purchase socks and other aspects. With the rapid development of computer appliance technology, CAD/CAM technologies are increasingly used in pattern design and process treatment for automatic hosiery machines, bringing great advancement to the traditional hosiery machine industry. However, traditional methods are still used in the existing hosiery machine CAD in terms of pattern and design plan. In particular, mapping is conducted by a stylist, and the design is reflected through drawings or other media and then translated into patterns. Moreover, existing pictures and photos are scanned by devices like scanners. The design is then modified and transformed into pattern files. With long design cycles, limited color choices, and monotonous graphics, such patterns have trouble meeting increasingly high requirements of people to pursue novelty and beauty and highlight personality [2-6]. Application of fractal geometry in pattern design in automatic hosiery machines can generate abundant patterns, but literature concerning the application of fractal geometry in computerized hosiery machines is limited. In this study, iterative algorithm, recursive algorithm, complex plane algorithm, and cellular automata algorithm are used. Abundant fractal graphics are created by a developed computer software through simple algorithm. Thus, complicated scenery graphics can be created simply [7-10], substantially enriching color variants in sock products and shortening pattern design cycles. 


\section{Integral structure design of the system}

The elements of the system interface include a title bar, menu bar, tool bar, work area, and status bar. With the typical style of a Windows appliance, the graphical operation interface is highly interactive (Figure 1).

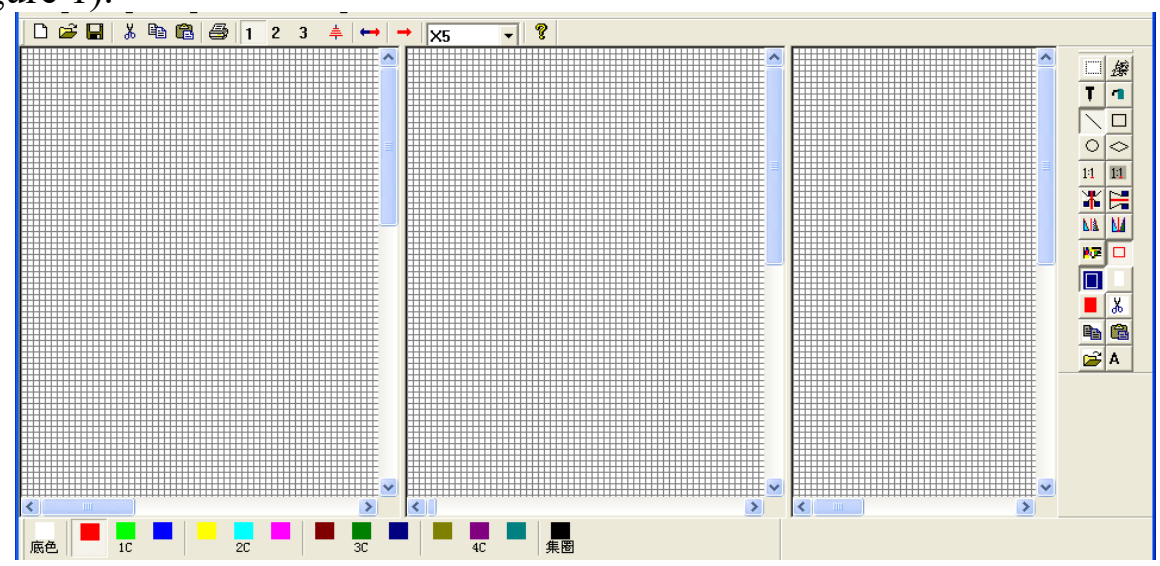

Figure 1 Diagram of the system interface

\subsection{Fractal graphics module of pattern of hosiery machine}

Many vivid and bizarre graphics can be plotted using the characteristics and description methods of fractal graphics. Fractal graphics can be drawn on the computer as long as certain rules are formulated. In this section, the "rules," which vary with different fractal models, are explained with respect to different models as follows.

\subsection{Fractal graphic models of iterated function system}

With the affinitive transformation as a frame, based on the fact that the entirety and locality of geometry objects have self-similar structures, the iterated function system (IFS) can build endless and changeable fractal graphics by iterating the overall morphology according to different affinitive transformations in a certain probability. This paper uses the deterministic algorithm, randomized iterative algorithm,[11] and improved IFS algorithm in the IFS to create fractal graphics based on iterative function theory. Among the three algorithms, the deterministic algorithm can plot fractal graphics according to six appropriate IFS codes (a, b, c, d, e, and f). The randomized iterative algorithm can plot fractal graphics according to seven appropriate IFS codes (a, b, c, d, e, f, and g). The improved IFS algorithm can generate new fractal graphics based on the above two IFS codes as long as one or two parameters are changed.

\subsection{Fractal graphics models of uniserial recursive system}

The following story is a common childhood tale. A long time ago, there was a mountain. On the mountain, there was a temple. In the temple, there was an old monk. The old monk told a young monk a story [12]. The old monk said, "A long time ago, there was a mountain. On the mountain, there was a temple. In the temple, there was an old monk. The old monk told a young monk a story. The old monk said, 'A long time ago, there was a mountain. On the mountain, there was a temple. In the temple, there was an old monk. The old monk told a young monk a story. The old monk said" This story is merely a last resort an adult uses when stuck by a child and has no other stories to tell. However, the story is a typical iterative process. Most fractal graphics can be accomplished 
with an iterative method. The iterative method it uses is realized through repeated use of its principle by function. In this study, the iterative fraction is to carry out iteration after the drawing function is executed. It has straight and smooth curve portions and looks more coordinated than graphics that are full of curves. For example, the tree curve is a curve created through iteration and repeated branching, which has appropriate complexity and equilibrium, giving people an incredible aesthetic feeling. Many fractal graphics are created through iteration, including a tree curve, segment recursive curve, round iterative curve, Mandala recursive curve, regular polygon recursive curve, and asteroid-shaped curve, based on different basic elements of recursion [13, 14]. The constitution methods of these curves are the same.

\subsection{Fractal graphics models of complex plane system}

A complex plane, which is slightly different from a regular plane, mainly adds a concept of imaginary number. The real number is represented by the $\mathrm{X}$ axis, and the imaginary number is represented by the $\mathrm{Y}$ axis. The creation process is as follows: assuming that the function or mapping $y=f(x)$ is one rule, if an initial value of $x$ is given, marked as $x 0$, then there is $y=f(x)$, and one value of $y$ can obtained, which is marked as $\mathrm{x} 1$, i.e., $\mathrm{x} 1=\mathrm{f}(\mathrm{x} 0)$, this $\mathrm{y}=\mathrm{x} 1$ is taken as a new value of $\mathrm{x}$, and then $y$ 's new value of $y=f(x 1)$ can be obtained, marked asx2, i.e., $x 2=f(x 1)$. The repeatedly iterated mathematic feedback process makes every output of the function or mapping as the next input, i.e., $\mathrm{xn}+1=\mathrm{f}(\mathrm{n}), \mathrm{n}=0,1,2 \ldots \ldots$, then a sequence of $\mathrm{x} 0, \mathrm{x} 1, \mathrm{x} 2, \ldots, \mathrm{xn}, \ldots$, can be obtained. This sequence is called an iterative orbit of $\mathrm{x} 0$. Well-known fractal sets are Mandelbrot set and Julia set. The mathematic expressions of both sets are similar, and they use two-dimensional iteration relations on the complex plane:

$$
Z \rightarrow Z^{2}+C
$$

\subsection{Fractal models of cellular automata system}

The constitutional rule of this system is simple. The first row of pixels is first drawn when the graphics are plotted. Subsequent pixel rows are based on the previous rows, and a value is obtained according to a certain computation rule to determine whether each pixel point is plotted or not. Then, a design pattern is formed. Parameters are required for the formulation of cell proliferation rules besides pattern width and pattern height in the plotting parameters of fractal graphics of cellular automata. The status of the first row of pixels in the fractal graphics is first achieved according to a certain rule, that is, the "living" and "death" status of each point in the first row, which indicates whether each point is drawn or not. Each subsequent row is plotted in turn based on the first row, and the fractal graphics are finally achieved.

\section{Potting of fractal pattern of the system}

Visual $\mathrm{C}++$ is a programming method and a graphics plotting function based on object-oriented thinking. This method can take each specific fraction as an object using fractal theories and computer graphics-related knowledge simultaneously, as well as abstract corresponding graphics categories from multiple fractal objects with similar properties and behaviors. Adopting objectoriented programming technologies can easily generate splendid and enriched fractal graphics. The plotting process is shown in Figure 2, and the fractal graphics obtained by computers is shown in Figure 3. 


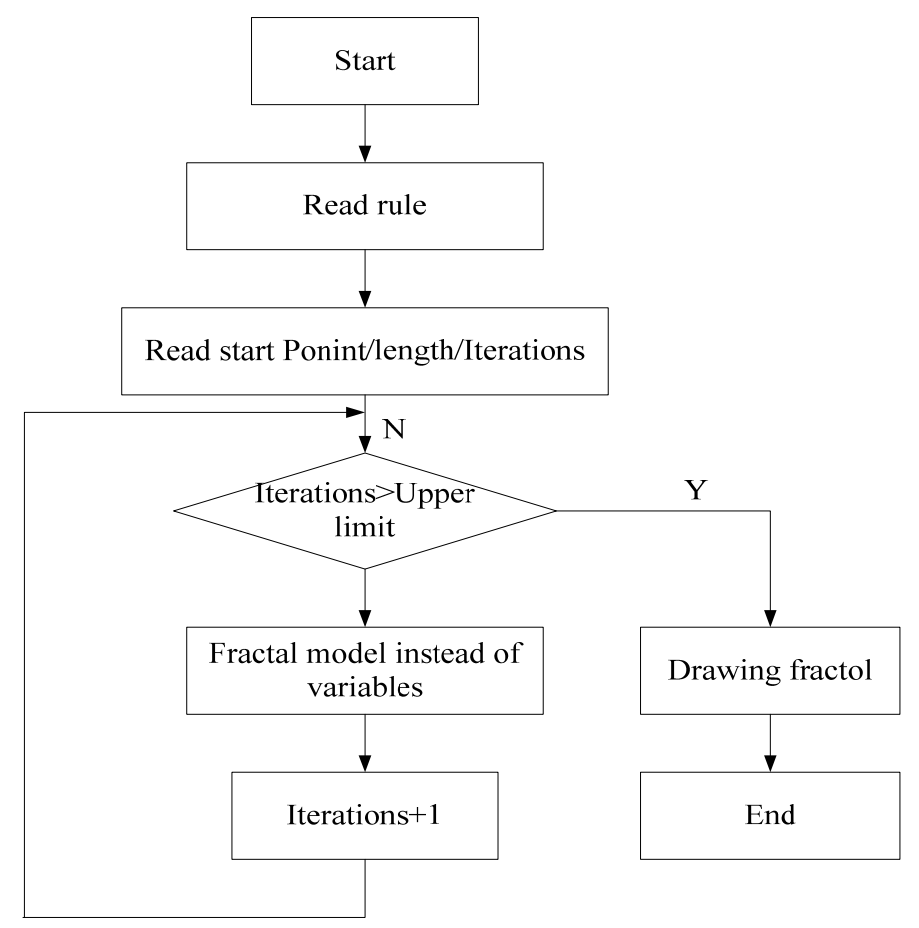

Figure 2 Plotting process of fractal graphics
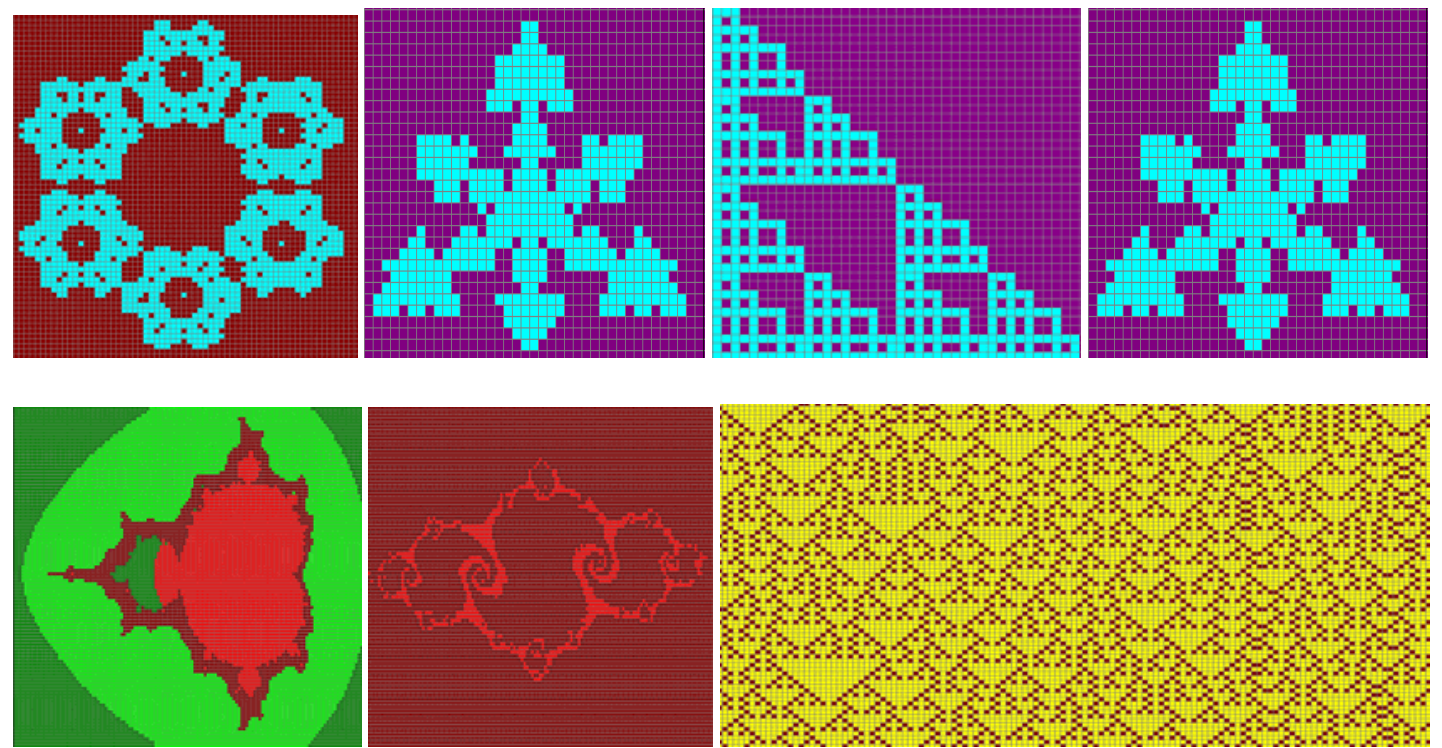

Figure 3 Fractal graphics

\section{Storage of fractal pattern files in the hosiery machine}

As fractal graphics are stored in a disk according to a universal graphic format, they cannot be directly used in knitting due to the failure of the universal picture format to be identified by the knitting system of the hosiery machine. Thus, another process is used. The graphic file format stored is first converted. Pattern information files are transformed into knitting files that can be identified by the hosiery machine and then input into the hosiery machine for knitting. In this paper, the file storage format of the hosiery machine is customized, and the generated fractal graphics are applied in the hosiery machine system designed by the author. 
As an example, the fractal pattern is 40 in width and 32 in height. The fractal graphics are shown in Figure 4.

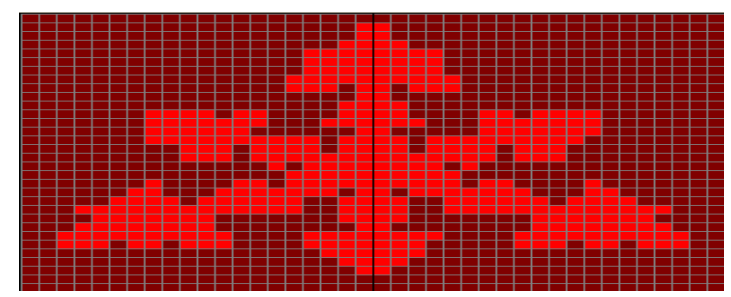

Figure 4 Fractal graphics(40 in width and 32 in height)

The hosiery machine data file for this fractal pattern is shown in Figure 5.

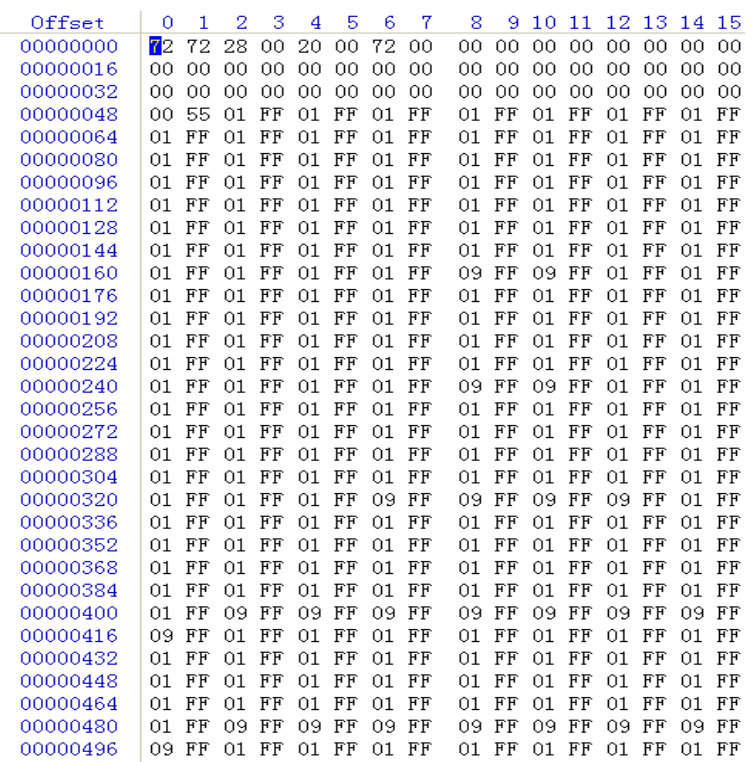

Figure 5 The hosiery machine data file

\section{Conclusion}

With nonlinear properties, fractal geometry can describe sceneries such as a cloud, snow, and tree in nature, and it has a wide application prospect. This study uses IFS, recursion fractal, complex plane fractal system, and cellular automata fractal models; obtains enriched fractal graphics; and successfully applies these fractal graphics in CAD design of patterns of sock products. Fractal graphics can improve design efficiency and enrich patterns. The research results can greatly increase CAD and CAM levels and productive efficiency in our country's sock manufacturing industry.

\section{References}

[1] Yang, X.H., Tang R.C. and Li D.G.(2004) Fractal Geometry - New Tool for Textile Design Development and Application. China Textile Leader, 6, 98-100.

[2] Yu, T.B., Cao Y.L. and Zhao Y.X.(2006) Design of a Pneumatic Pattern Embroidering Component Used in Sock Weaving Machine. Chinese Hydraulics \& Pneumatics, 3, 53-54.

[3] Mermelstein, S.P., Roberts, K. and Baggott, A.(1997) Bringing New Products to the Market. Wire Knitting Machines Case Study. Factory 2000 - The Technology Exploitation Process, Fifth International Conference on (Conf. Publ. No. 435). 2-4 April, 257 -262. 
[4] Power, E. J, Dias T.(2003) Knitting of Electroconductive Yarns. Eurowearable, IEE, 55 -60.

[5] Yan, X.H.(2004) Visualization of Fractal Information and Its Expression in Textile Art. Master Thesis. Suzhou: Suzhou University. 9-10.

[6] Zhao, Z.Y.(2002) Knitting. Xi'an: Northwestern Polytechnical University Press. 268-269.

[7] Qi, X.H., Sun, S.S., Lin, M.L. and Qiao, X.L.(2006) Artificial Fur Knitting Machine Auto-Control System. Intelligent Control and Automation, WCICA 2006. The Sixth World Congress on. 21-23 June,2, 8006-8010.

[8] Catarino, A., Rocha, A.M., Monteiro, J.L. and Soares, F.(2004) Surveillance and Control of the Yarn Input Tension on Circular Weft Knitting Machines: New Approaches. Industrial Technology, IEEE ICIT '04. 2004 IEEE International Conference on. 8-10 Dec. 2004,3(3), 1642-1648.

[9] Zaharieva-Stoyanova, E.(2006) Algorithm for Computer Aided Design Curve Shape Form Generation of Knitting Patterns. Automation, Quality and Testing, Robotics, 2006 IEEE International Conference on,1, 327-331.

[10] Catarino, A., Rocha, A., Monteiro, J.L. and Soares, F.(2004) Techniques for Unveiling Faults During, Knitting, Production. Industrial Electronics, 2004 IEEE International Symposium on.4-7 May,1(1), 389-394.

[11] Sun W., Chen J.C.(2001) The Simple Way of Using Iteration Function System to Obtain the Fractal Graphics. Journal of Engineering Graphics, 3, 109-113.

[12] Sun B.W.(2004) Fractal algorithm and program design (Visual C++realization). Beijing: Science Press, 1012.

[13] Pan J.G., Ai Z.Y.(1998) Fractal Art Program Design. Nanjing: Nanjing University Press, 71-98.

[14] Zhang Y.H., Li X.L., Wang Z.W. and Wang Y.J.(2000) Fractal Simulation of Natural Tree's Appearance. Computer Engineering, 9, 112-114.

[15] Zhang L.S., Zhang Y.S.(2000) Research on OO Method Based Fractal Graphics Generation. Computer Engineering, 5, 28-29. 